INPLASY

PROTOCOL

To cite: Li et al. Effect of cardiac shock wave therapy on adverse cardiovascular event for patients with coronary artery disease: an updated systematic review and metaanalysis. Inplasy protocol 202210103. doi: 10.37766/inplasy2022.1.0103

Received: 20 January 2022

Published: 20 January 2022

Corresponding author: Peng Li

lipbenzi@126.com

Author Affiliation: Beijing Hospital, National Center of Gerontology

Support: Beijing Hospital Clinical Rese.

Review Stage at time of this submission: Preliminary searches.

Conflicts of interest: None declared.

\section{Effect of cardiac shock wave therapy on adverse cardiovascular event for patients with coronary artery disease: an updated systematic review and meta-analysis}

Li, P1; Jia, N2; Liu, B33 He, Q4

Review question / Objective: We have previously demonstrated that cardiac shock wave therapy (CSWT) effectively improves myocardial perfusion in patients with coronary artery disease (CAD). In this study, we want to address whether CSWT could decrease the risk of adverse cardiovascular events in CAD patients unsuitable for revascularization.

Eligibility criteria: Trials are considered eligible if they meet these criteria: (1) patients included are diagnosed as refractory angina or ischemic heart failure; (2) the study i a randomized controlled trial (RCT) or a prospective cohort study; (3) intervention consisted of CSWT; (4) patients in the control group are treated with optimal medical therapy, (5)the primary outcome of interest is rate of MACE. Exclusion criteria were (1) patients with acute myocardial infarction, (2) repeated CSWT, (3) with coronary artery revascularization, (4) without primary outcome, (5) retrospective study, and (6)duplicated data.

INPLASY registration number: This protocol was registered with the International Platform of Registered Systematic Review and Meta-Analysis Protocols (INPLASY) on 20 January 2022 and was last updated on 20 January 2022 (registration number INPLASY202210103).

\section{INTRODUCTION}

Review question / Objective: We have previously demonstrated that cardiac shock wave therapy (CSWT) effectively improves myocardial perfusion in patients with coronary artery disease (CAD). In this study, we want to address whether CSWT could decrease the risk of adverse cardiovascular events in CAD patients unsuitable for revascularization.

Rationale: Several studies have demonstrated that cardiac shock wave therapy (CSWT) is an efficient option for refractory angina or ischemic heart failure in animal models and human patients. Our previous studies have indicated that CSWT 
exerts a protective cardiac effect through inhibiting ischemia/hypoxia-induced H9c2 myoblast cell apoptosis and promoting cardiomyocyte autophagy during hypoxia. Therefore, CSWT not only improves myocardial perfusion, cardiac function, and myocardial ischemia symptoms, but also ameliorates quality of life in patients with CAD. Furthermore, the effect of CSWT on hard endpoints such as mortality, myocardial infarction and rehospitalization has not been evaluated in any metaanalyses.

Condition being studied: The treatment of refractory angina or ischemic heart failure is challenging, as those patients experience angina even with minimal activity or at rest. Therefore, these individuals suffer a severely increased risk of rehospitalization for frequent angina, myocardial infarction and even death, although the risk of mortality is similar to that for stable coronary artery disease. Cardiac shock wave therapy (CSWT) has been reported to potentially promote myocardial blood flow, reduce angina symptoms, and increase cardiac function. Hence, CSWT may potentially decrease the rate of rehospitalization as a consequence of angina symptoms. In a clinical study of CSWT for 45 RA patients, Yang et al. found that CSWT markedly decreased the rate of rehospitalization for myocardial ischemic symptoms at 6-month follow-up in comparison with a control group $(20.0 \%$ versus $55.0 \%, P<0.05)$. In another study conducted in China with 12 months of follow-up, old myocardial infarction patients in the control group were associated with a significantly higher rate of rehospitalization because of CAD when compared with patients in the regular CSWT group $(56.0 \%$ vs $21.8 \%)$ or those in the expanding scope CSWT group $(56.0 \%$ vs $16.7 \%$ ). However, previous evidence is limited to mainly small-sized, single-arm, low- to moderate-quality, single-center studies with mixed results.

\section{METHODS}

Search strategy: We will search, with no time restrictions, the following databases for relevant English language literature: PubMed (MEDLINE), the Cochrane Central Register of Controlled Trials (CENTRAL) and Web of Science.The search string will be built as follows: (coronary artery disease OR CAD) AND (coronary heart disease OR CHD) AND (cardiac shock wave therapy OR shock wave) AND (mortality OR death OR rehospitalization OR myocardial infarction OR randomized trial OR prospective cohort study). The electronic database search will be supplemented by a manual search of the reference lists of included articles.

Participant or population: Coronary artery disease.

Intervention: Cardiac shock wave therapy + optimal medical therapy.

\section{Comparator: Optimal medical therapy.}

Study designs to be included: Randomized clinical study or prospective cohort study.

Eligibility criteria: Trials are considered eligible if they meet these criteria: (1) patients included are diagnosed as refractory angina or ischemic heart failure; (2) the study i a randomized controlled trial (RCT) or a prospective cohort study; (3) intervention consisted of CSWT; (4) patients in the control group are treated with optimal medical therapy, (5)the primary outcome of interest Is rate of MACE. Exclusion criteria were (1) patients with acute myocardial infarction, (2) repeated CSWT, (3) with coronary artery revascularization, (4) without primary outcome, (5) retrospective study, and (6)duplicated data.

Information sources: MEDLINE (source, PubMed), EMBASE, the Cochrane Controlled Clinical Trials Register Database, the ClinicalTrials.gov website, and the Chinese SinoMed Database.

Main outcome(s): Primary outcome was the rate of major adverse cardiovascular event (the composite outcome of mortality, coronary artery revascularization, myocardial infarction and rehospitalization). 
Quality assessment / Risk of bias analysis: For randomized clinical study, the Cochrane Collaboration method is used for quality assessment. For prospective cohort studies, we use the Newcastle-Ottawa scale (NOS) to evaluate the methodological quality. Heterogeneity was examined by the I2 statistic and the chi-squared test. A value of $12>50 \%$ was considered a substantial level of heterogeneity. Publication bias was assessed quantitatively using Egger's regression test $(P \leq 0.10)$ [18] and qualitatively by visual inspection of funnel plots of the logarithm of RRs versus their standard errors.

Strategy of data synthesis: Results were analyzed quantitatively with STATA $\mathbf{1 4 . 0}$ software (Stata Corp, College Station, TX, USA) using the fixed- and random-effects (DerSimonian and Laird random-effects) models. We calculate the pooled relative risk (RR) for dichotomous outcomes and the standard mean difference (SMD) or weighted mean difference (WMD) for continuous data with $95 \%$ confidence interval (Cl).

Subgroup analysis: Once heterogeneity was noted, between-study sources of heterogeneity were investigated using subgroup analysis by stratifying original estimates according to study characteristics (age, male, previous conditions, heart function, follow-up duration, study design).

Sensitivity analysis: Sensitivity analyses were conducted to determine the influence of individual trials on the overall pooled results (MACE).

Language: No restriction.

Country(ies) involved: China.

Keywords: Cardiac shock wave therapy; Refractory angina; Ischemic heart failure; Major adverse cardiac events; Rehospitalization; Meta-analysis.

Dissemination plans: We want to publish it in JAMA Open Work and communicate it at the Great Wall International Congress of
Cardiology 2022 and Asia Heart Society Congress 2022.

Contributions of each author:

Author 1 - Peng Li - The author drafted the manuscript.

Author 2 - Na Jia - The author provided statistical expertise.

Author 3 - Bing Liu - The author contributed to the development of the selection criteria, and the risk of bias assessment strategy.

Author 4 - Qing He - The author read, provided feedback and approved the final manuscript. 